Progress of Theoretical Physics, Vol. 89, No. 1, January 1993

\title{
Schwinger-Dyson Equation in Three-Dimensional Simplicial Quantum Gravity
}

\author{
Hirosi OOGURI \\ Research Institute for Mathematical Sciences, Kyoto University, Kyoto 606-01 \\ and \\ Lyman Laboratory of Physics, Harvard University, Cambridge, MA 02138
}

(Received October 11, 1992)

\begin{abstract}
We study the simplicial quantum gravity in three dimensions. Motivated by Boulatov's model which generates a sum over simplicial complexes weighted with the Turaev-Viro invariant, we introduce boundary operators in the simplicial gravity associated to compact orientable surfaces. An amplitude of the boundary operator is given by a sum over triangulations in the interior of the boundary surface. It turns out that the amplitude solves the Schwinger-Dyson equation even if we restrict the topology in the interior of the surface, as far as the surface is no-degenerate. We propose a set of factorization conditions on the amplitudes which singles out a solution associated to triangulations of $S^{3}$.
\end{abstract}

\section{§ 1. Introduction}

In order to understand a quantum theory of gravity, we need to clarify what we mean by integrations over Riemannian metrics on a $d$-dimensional differentiable manifold $M$. One of the possibilities proposed by Regge ${ }^{1)}$ is to discretize the integrals and replace them by sums over smooth triangulations on $M$. In this approach, each triangulation $T$ specifies a Riemannian metric on $M$ in the following way. We impose that an interior of each $d$-simplex in $T$ is flat Euclidean and that the curvature has a support only on $(d-2)$-simplexes. Moreover all the edges in $T$ are regarded as being straight and having the same geodesics length $a$. (There is another version of the theory where the edges are allowed to have different lengths, ${ }^{2)}$ but we do not examine this possibility here.). These conditions are enough to define a Riemannian metric associated to the triangulation $T$ of $M$. It is reasonable to expect that any metric on $M$ can be approximated in this way by using sufficiently large number of simplexes and by taking the size $a$ of each simplex to be infinitesimally small. It is then important to know what types of triangulations give relevant contributions to the summations. If smooth triangulations with infinitely many simplexes dominate the summations, it would make sense to define the integrals over metrics by the sums over triangulations. In two dimensions, this program has worked rather well and we have learned quite a lot about two-dimensional quantum gravities from this approach for the last few years. The purpose of this paper is to study the three-dimensional case.

Let us be more specific about what we mean by the sums over triangulations. In order to quantize the Einstein gravity, we would like to perform integrals over metrics $g_{\mu \nu}$ weighted with the exponential of the action given by $S[g]=\Lambda \int d^{d} x \sqrt{g}+(1 / G) \int d^{d} x$ $\sqrt{g} R$ where $R$ is the scalar curvature. Suppose that the metric $g$ is associated to 
some triangulation $T$ of $M$. Since each $d$-simplex has the same volume proportional to $a^{d}$, the total volume $\int d^{d} x \sqrt{g}$ of $M$ should be proportional to $a^{d} n_{d}(T)$ where $n_{i}(T)$ $(i=0,1, \cdots, d)$ is the number of $i$-simplexes in $T$. The integral of the scalar curvature $R$ on the other hand is expressed in the cases $d=2$ and 3 as follows. When $d$ $=2$, the curvature has a support on the vertices of $T$. Since the deficit angle around each vertex $v$ is given by $\left(2 \pi-(\pi / 3) \tau_{\Delta}(v)\right)$ where $\tau_{\Delta}(v)$ is the number of triangles containing $v$, we obtain

$$
\int d^{2} x \sqrt{g} R \sim \sum_{v: \text { vertices }}\left(1-\frac{1}{6} \tau_{\Delta}(v)\right)=n_{0}(T)-\frac{1}{2} n_{2}(T)
$$

Especially when the manifold $M$ is closed, $3 n_{2}(T)=2 n_{1}(T)$ since every edge on $T$ is shared by exactly two triangles. In this case, the right-hand side in the above becomes $\chi=n_{0}(T)-n_{1}(T)+n_{2}(T)$, the Euler number of $M$. When $d=3$, the curvature is concentrated on the edges of $T$ and is characterized by the deficit angle around each edge $e$ given by $\left(2 \pi-\cos ^{-1}(1 / 3)\right)$ where $\tau_{t}(e)$ denotes the number of tetrahedra containing $e$. Since each edge has the geodesic length $a$, the integral of the scalar curvature is given by

$$
\int d^{3} x \sqrt{g} R \sim a \sum_{e: \text { edges }}\left[2 \pi-\cos ^{-1}\left(\frac{1}{3}\right) \tau_{t}(e)\right]=a\left[2 \pi n_{1}(T)-6 \cos ^{-1}\left(\frac{1}{3}\right) n_{3}(T)\right] .
$$

Therefore, for both $d=2$ and 3 , the action $S[g]$ is expressed as a linear combination of $n_{d}(T)$ and $n_{d-2}(T)$.

$$
S[g]=-\kappa n_{d-2}(T)+\beta n_{d}(T) .
$$

The partition function of the simplicial quantum gravity is then defined by

$$
Z_{d}=\sum_{T} \frac{1}{C(T)} e^{\kappa n_{d-2}(T)-\beta n_{d}(T)}
$$

where the sum is over all possible triangulations of $M$, and the factor $C(T)$ is the order of a symmetry of $T$ if any. This factor is included in order to take into account the volume factor of the diffeomorphism group. First of all, we need to know if this summation is convergent at all. Since $Z_{d}$ can be written as

$$
Z_{d}=\sum_{n_{d}, n_{d-2}=0}^{\infty} Z_{n_{d}, n_{d-2}}(M) e^{\kappa n_{d-2}-\beta n_{d}}
$$

where $Z_{n_{d, n} n_{d-2}}(M)$ is a number of triangulations of $M$ with given values of $n_{d}$ and $n_{d-2}$ weighted with the factor $C(T)$, we would like to know the asymptotic behavior of $Z_{n_{d}, n_{d-2}}(M)$ for large values of $n_{d}$ and $n_{d-2}$. In two dimensions, $n_{2}$ and $n_{0}$ are not independent and they are related as $n_{0}=(1 / 2) n_{2}+\chi$. Especially when $\chi=2$ (i.e., $M \simeq$ $S^{2}$ ), the exact asymptotic behavior of $Z_{n_{2}, n_{0}}$ is known from the combinatorial study of Ref. 3) as

$$
Z_{n_{2}, n_{0}}\left(S^{2}\right) \sim n_{2}{ }^{-7 / 2} e^{\beta_{c} n_{2}}, \quad\left(n_{2} \rightarrow \infty, n_{0}=\frac{1}{2} n_{2}+2\right)
$$




$$
e^{\beta_{c}}=\frac{2^{4}}{3^{3 / 2}} .
$$

The sum over triangulations can also be evaluated by the matrix model, ${ }^{4)}$ and the above formula for $S^{2}$ is reproduced by the sum over one-particle irreducible vacuum diagrams ${ }^{4)}$ in the large- $N$ limit of the matrix model. ${ }^{5) \sim 7)}$ (The sum over connected vacuum diagrams also gives a similar formula with a different value of $\beta_{c} ; e^{\beta_{c}}=2 \cdot 3^{3 / 4}$.) When $M$ is a surface of higher genus $(\chi \leq 0)$, subleading corrections ${ }^{8)}$ to the large- $N$ matrix model give ${ }^{9)}$

$$
Z_{n_{2} n_{0}}(M) \sim n_{2}{ }^{-(5 / 4) \chi-1} e^{\beta_{c} n_{2}} . \quad\left(n_{2} \rightarrow \infty, n_{0}=\frac{1}{2} n_{2}+\chi\right)
$$

Therefore the contribution to $Z_{d=2}$ from $T$ with many triangles is expressed as

$$
Z_{d=2} \sim e^{\kappa \chi} \sum_{n_{2}} n_{2}{ }^{-(4 / 5) \chi-1} e^{-\left(\tilde{\beta}-\beta_{c}\right) n_{2}} \sim\left\{\begin{array}{cl}
\left(\tilde{\beta}-\beta_{c}\right)^{(5 / 4) x} & \text { if } \chi \neq 0 ; \\
\log \left(\frac{1}{\tilde{\beta}-\beta_{c}}\right) & \text { if } \chi=0,
\end{array}\right.
$$

where $\tilde{\beta}=\beta-(1 / 2) \kappa$, and it is convergent for $\tilde{\beta}>\beta_{c}$. Moreover as $\tilde{\beta}$ approaches the critical value $\beta_{c}$, the contributions from triangulations with $n_{2} \gg 1$ become more and more significant for $Z_{d=2}$ with $\chi \leq 0$ and for $\partial_{\beta}^{3} Z_{d=2}$ with $\chi=2$. Thus we have a good reason to believe that the program of the simplicial quantum gravity works well in two dimensions. Indeed this approach has turned out to be quite successful and its validity has been confirmed by comparisons of the matrix model computations with the results of the continuum field theories. ${ }^{10) \sim 16)}$

Recently several numerical results have been reported on the three-dimensional simplicial quantum gravity. ${ }^{17) ~ 21)}$ Their results seem to be compatible with the following bound on the number of triangulations of $S^{3}$ :

$$
Z_{n_{3}}\left(S^{3}\right)=\sum_{n_{1}} Z_{n_{3} n_{1}}\left(S^{3}\right)<C e^{\beta_{c} n_{3}}, \quad\left(n_{3} \rightarrow \infty\right)
$$

for some constants $C$ and $\beta_{c}$. In three dimensions, the Euler number $\chi=n_{0}-n_{1}+n_{2}$ $-n_{3}$ is zero for any triangulation of a closed manifold. We also have $n_{2}=2 n_{3}$ which follows from the fact that any triangle in $T$ is shared by exactly two tetrahedra. Therefore $n_{3}=n_{1}-n_{0}$, and $n_{3}$ should always be less than $n_{1}$. On the other hand, since each edge in $T$ belongs to at least one tetrahedra, $n_{1}$ must be less than or equal to $6 n_{3}$. Namely $n_{3}<n_{1} \leq 6 n_{3}$. Thus, if the exponential bound (3) is indeed correct, the sum over triangulations in Eq. (1) would be bounded as

$$
Z_{d=3} \leq \sum_{n_{3}, n_{1}} Z_{n_{3} n_{1}} e^{-\left(\beta-6_{\kappa}\right) n_{3}}<C \sum_{n_{3}} e^{-\left(\beta-6 \kappa-\beta_{c}\right) n_{3}}
$$

for $\kappa \geq 0$ and

$$
Z_{d=3}<\sum_{n_{3}, n_{1}} e^{-(\beta-\alpha) n_{3}}<C \sum_{n_{3}} e^{-\left(\beta-\kappa-\beta_{c}\right)}
$$

for $\kappa<0$. The sum (1) would then be convergent at least for $\beta-6 \kappa>\beta_{c}(\kappa \geq 0)$ and for $\beta-\kappa>\beta_{c}(0>\kappa)$. It is thus important to develop an analytical tool to examine the behavior of $Z_{n_{3}}\left(S^{3}\right)$. 
In two dimensions, the matrix model ${ }^{4)}$ has been found useful to study the simplicial quantum gravity, and there have been some attempts to extend it to three dimensions. ${ }^{22) ~ 24)}$ In this so-called tensor model, one considers an integral over rank-three tensors rather than matrices, and the perturbative expansion of the integral generates simplicial complexes, i.e., collections of tetrahedra whose boundary triangles are pairwisely identified. However the collections of tetrahedra generated in this model are not necessarily triangulations of smooth manifolds. In fact their Euler numbers are not always zero, but in general non-negative integers. ${ }^{22}$

The basic reason for the failure of the tensor model is that the model does not contain as many parameters as one needs to distinguish various topologies in three dimensions. In two dimensions, a topology of a closed orientable surface is characterized by the number of handles, and two parameters in the model are enough to control both the topology and the volume $n_{2}$ of the surface. The matrix model indeed has two parameters, the size $N$ of the matrix and the coupling constant $g$. In three dimensions, on the other hand, we need at least three parameters to control three independent variables $n_{3}, n_{1}$ and $\chi=n_{0}-n_{1}+n_{2}-n_{3}$. The simplest version of the tensor model has only two parameters, the size $N$ of the tensor and the coupling constant, and it is not possible to force $\chi$ to be zero.

Recently, Boulatov proposed an improved version of the tensor model ${ }^{25)}$ by incorporating a gauge symmetry of a finite group or a quantum group. If one takes the gauge group to be a finite group $G$, the order of $G$ couples to the Euler number of the simplicial complex. For example, when $G$ is the cyclic group $Z_{p}$, the partition function of the model is expressed as a sum over simplicial complexes $T$ weighted with a factor $p^{x(T)}\left|H_{1}\left(T, Z_{p}\right)\right|$, where $\left|H_{1}\left(T ; Z_{p}\right)\right|$ is the order of the first cohomology group of $T$ with coefficients in $Z_{p}$. In three dimensions, the necessary and the sufficient condition for a simplicial complex $T$ to be a manifold is $\chi(T)=0$, and otherwise $\chi(T)>0$. Thus, if one could make sense of the limit of $p \rightarrow 0$, the perturbative expansion of the model in this limit would be dominated by the sum over simplicial manifolds with $\chi(T)=0$.

The $p \rightarrow 0$ limit of the $Z_{p}$ model not only imposes $\chi=0$ but gives an additional condition on topologies of simplicial manifolds, i.e., the first and the second homologies of the manifolds are restricted to be trivial..$^{25}$. Without any condition on the topology, the number of three-dimensional simplicial manifolds with a given number $n_{3}$ of tetrahedra grows at least factorially in $n_{3}{ }^{22)}$ and the sum over simplicial manifolds becomes divergent. Thus one needs to impose restrictions on the topologies. It is not clear if the conditions on the homologies are enough to tame the factorial growth since there are many topologies in three dimensions with the same homologies as those of $S^{3}$. If not, the sum over triangulations generated by the $Z_{p}$ model would still be divergent, and we would need to develop a mechanism to impose further restrictions on the topologies.

In this paper, we examine Boulatov's model associated to the quantum group $S U_{q}(2)$. In this model, the sum over simplicial complexes is weighted with $\Lambda_{q}^{\chi(T)} I_{q}(T)$ where 


$$
\Lambda_{q}=-\frac{2(k+2)}{\left(q^{1 / 2}-q^{-1 / 2}\right)^{2}}, \quad q=e^{2 \pi i / k+2)}
$$

and $I_{g}(T)$ is the Turaev-Viro invariant. ${ }^{26)}$ We define boundary operators of the model associated to compact orientable triangulated two-dimensional surfaces, and derive the Schwinger-Dyson equation for the amplitudes of the operators. It turns out that the Schwinger-Dyson equation takes the same form for any value of $q$ when the surfaces involved are non-degenerate. Since the amplitude of the boundary operator in the Boulatov model is expressed as a sum over triangulations in the interior of the boundary surface weighted with $\Lambda_{q}{ }^{x} I_{q}$ depending on $q$, the independence of the Schwinger-Dyson equation on $q$ suggests that we can restrict the topology of $T$ in the interior of the surface without spoiling the equation. Indeed we find that, associated to an arbitrary closed orientable three-dimensional manifold, one can construct a solution to the equation. In order to single out a solution associated to triangulations of $S^{3}$, we introduce a set of factorization conditions for degenerate surfaces with which the equation can be solved uniquely and inductively.

In the case of the two-dimensional simplicial gravity, the Schwinger-Dyson equation can be derived from purely combinatorial considerations without using the matrix model. This is also true in the three-dimensional model studied here. However, in order to take into account the symmetry factor to each graph, it is more convenient and transparent to employ the perturbative expansion of the Boulatov model.

This paper is organized as follows. In $\S 2$, we briefly describe the SchwingerDyson equation in the large- $N$ matrix model and its combinatorial meaning. We then define Boulatov's model in $\S 3$ and examine its properties. The boundary operator is defined in $\S 4$, and the.Schwinger-Dyson equation is derived. In $\S 5$, we show that there is a solution to the equation associated to each closed orientable three-dimensional manifold. The factorization conditions on the amplitude which characterize the solution associated to $S^{3}$ are defined in $\S 6$. The Schwinger-Dyson equation combined with the factorization conditions is shown to have a unique solution.

\section{§2. Schwinger-Dyson equation in the matrix model}

Let $\left(M_{i j}\right)$ be an $N \times N$ hermitian matrix and consider the following integral:

$$
Z=\int[d M] \exp (-S(M)),
$$

where

$$
S(M)=\frac{1}{2} \operatorname{tr} M^{2}-\frac{g}{3 \sqrt{N}} \operatorname{tr} M^{3}
$$

by expanding the integral (4) in powers of $g, Z$ is expressed as a sum over orientable closed trivalent graphs, each of which is dual to a triangulation of an orientable closed (but not necessarily connected) surface. A sum over closed and connected simplicial 
manifolds is obtained by taking the logarithm of $Z$ as

$$
\log Z=\sum_{T} \frac{1}{C(T)}\left(\frac{g}{\sqrt{N}}\right)^{n_{2}(r)} N^{n_{0}(T)}=\sum_{T} \frac{1}{C(T)} g^{n_{2(T)}} N^{\chi(T)} .
$$

Especially the sum over triangulations of $S^{2}$ is given by the leading term in the large$N$ limit of $\log Z$.

The basic quantity we examine in this section is the expectation value of $\operatorname{tr} M^{n}$ at the large- $N$.

$$
u_{n}=\lim _{N \rightarrow \infty} \frac{1}{N^{(1 / 2) n+1}}\left\langle\operatorname{tr} M^{n}\right\rangle=\lim _{N \rightarrow \infty} \frac{1}{N^{(1 / 2) n+1}} \cdot \frac{1}{Z} \int[d M] \operatorname{tr} M^{n} \exp (-S(M)) .
$$

The power of $N$ is multiplied to $\left\langle\operatorname{tr} M^{n}\right\rangle$ so that $u_{n}$ gives a finite value in the limit of $N \rightarrow \infty$. The Schwinger-Dyson equation for $u_{n}^{27), 28}$ is derived from

$$
\begin{aligned}
0 & =\frac{1}{Z} \int[d M] \frac{\partial}{\partial M_{i j}}\left[\left(M^{n-1}\right)_{i j} \exp (-S(M))\right] \\
& =-\left\langle\operatorname{tr} M^{n}\right\rangle+\frac{g}{\sqrt{N}}\left\langle\operatorname{tr} M^{n+1}\right\rangle+\sum_{k=0}^{n-2}\left\langle\operatorname{tr} M^{k} \operatorname{tr} M^{n-k-2}\right\rangle .
\end{aligned}
$$

Here the integration by part is justified in each term in the perturbative expansion in $g$. In the limit of large- $N$, the last term on the right-hand side of Eq. (5) factorizes as

$$
\frac{1}{N^{(1 / 2) k+1}} \frac{1}{N^{(1 / 2)(n-k-2)+1}}\left\langle\operatorname{tr} M^{k} \operatorname{tr} M^{n-k-2}\right\rangle=u_{k} u_{n-k-2}+O\left(\frac{1}{N^{2}}\right)
$$

since only the planar graphs contribute. Thus we obtain a recurrence relation for $u_{n}$ as

$$
u_{n}=g u_{n+1}+\sum_{k=0}^{n-2} u_{k} u_{n-k-2} .
$$

This is the Schwinger-Dyson equation in the large- $N$ limit of the matrix model.

This equation has the following simple graphical interpretation. The expectation value $u_{n}$ can be expressed as a sum over planar trivalent graphs with $n$ external legs. The graphs are not necessarily connected, but they do not contain a closed disconnected part because of the factor $1 / Z$ in $u_{n}$. Let us take one of the graphs $T$ in the summation and pay attention to one of the external legs on it. The leg should be either connected to a trivalent vertex in $T$ or to another external leg. In the former case, the rest will be a graph with $(n+1)$ external legs. If on the other hand the leg is connected to another external leg, the line connecting these two legs will separate the original planar graph into two disconnected graphs with $k$ and $(n-k-2)$ external legs $(k=1,2, \cdots, n-2)$. The two terms on the right-hand side of the Schwinger-Dyson equation correspond to these two possibilities.

It is also straightforward to show that the Schwinger-Dyson equation completely determines the power series expansion of $u_{n}$ in $g$. By substituting

$$
u_{n}(g)=\sum_{n_{2}=0}^{\infty} u_{n, n_{2}} g^{n_{2}}
$$


into Eq. (6), we obtain a recurrence relation with respect to $n_{1}$ and $n_{2}$ as

$$
u_{n, n_{2}}=u_{n+1, n_{2}-1}+\sum_{k=0}^{n-2} \sum_{s=0}^{n_{2}} u_{k, s} u_{n-k-2, n_{2}-s}
$$

Since $u_{0}(g)$ is normalized to be 1 , the intial condition of the recurrence relation is $u_{0, n_{2}}$ $=\delta_{0, n_{2}}$, with which $u_{n, n_{2}}$ is determined inductively by solving (7) following the bibliographic ordering: $\left(n, n_{2}\right)>\left(n^{\prime}, n_{2}^{\prime}\right)$ if $n_{2}>n_{2}^{\prime}$ or $n_{2}=n_{2}^{\prime}, n>n^{\prime}$.

Since the nonlinear term in the Schwinger-Dyson equation (6) is the convolution of the $u_{n}$ 's, it can be transformed into the integral equation

$$
-1+g \lambda^{2}=P \int \frac{d \lambda^{\prime} \rho\left(\lambda^{\prime}\right)}{2 \pi \frac{\lambda^{\prime}}{\lambda-}}
$$

where

$$
u_{n}=\int d \lambda \rho(\lambda) \lambda^{n}
$$

and $P$ denotes the principal part of the integral. This equation can be solved using the technique of the Riemann-Hilbert correspondence ${ }^{4)}$ as

$$
\begin{aligned}
\rho(\lambda) & =\frac{1}{\pi}\left[1+\frac{1}{2} g(a+b)+g \lambda\right] \sqrt{(\lambda-a)(b-\lambda)} \quad(a \leq \lambda \leq b) \\
& =0, \quad(\lambda<a \text { or } b<\lambda)
\end{aligned}
$$

where $a$ and $b$ are determined by

$$
g(b-a)^{2}+2(a+b)[2+g(a+b)]=0, \quad(b-a)^{2}[1+g(a+b)]=1 .
$$

We can also compute the partition function (4) itself from the solution to the Schwinger-Dyson equation. By using the relation between $u_{3}$ and $Z$,

$$
u_{3}=\frac{1}{N^{2} \sqrt{N}}\left\langle\operatorname{tr} M^{3}\right\rangle=\frac{3}{N^{2}} \frac{1}{Z} \frac{\partial Z}{\partial g}
$$

we obtain

$$
Z(g)=\mathcal{Z}(0) \exp \left(\frac{N^{2}}{3} \int_{0}^{g} d g^{\prime} u_{3}\left(g^{\prime}\right)\right)
$$

The approach described here using the Schwinger-Dyson equation is in fact equivalent at the large- $N$ to the standard computation ${ }^{4), 8}$ using the eigenvalues $\lambda_{i}(i=1, \cdots, N)$ of $M$. By inverting the integral transformation (9), we can express $\rho(\lambda)$ in terms of $u_{n}$ as

$$
\begin{aligned}
\rho(\lambda)=\int \frac{d k}{2 \pi} e^{-i k \lambda} \sum_{n=0}^{\infty} \frac{1}{n !} u_{n} \lambda^{n} & =\int \frac{d k}{2 \pi} e^{-i k \lambda}\left\langle\frac{1}{N} \operatorname{tr} \exp \left(i k \frac{M}{\sqrt{N}}\right)\right\rangle \\
& =\sum_{i=1}^{N} \frac{1}{N}\left\langle\delta\left(\lambda-\frac{\lambda_{i}}{\sqrt{N}}\right)\right\rangle
\end{aligned}
$$

Namely $\rho(\lambda)$ is the density of the eigenvalues of $M$. 


\section{§3. Boulatov's model}

In this section, we describe the model due to Boulatov ${ }^{25)}$ and examine its properties. Let us consider the following integral:

$$
Z_{q}=\int[d M] \exp (-S(M))
$$

with $S(M)$ given by

$$
\begin{aligned}
& S(M)=\frac{1}{6} \sum_{a_{1}, a_{2}, a_{3}=1}^{N} \sum_{\left\{j_{1}, j_{2}, j_{3}\right\}} \sum_{\left\{-j_{k} \leq m_{k} \leq j_{k}\right\}}\left|M_{m_{1} m_{2} m_{3}}^{j_{1} j_{2} j_{3} \dot{a}_{1} a_{1} a_{2} a_{3}}\right|^{2} \\
& -\frac{g \Lambda_{q}}{12} \sum_{a_{1}, \cdots a_{6}=1}^{N} \sum_{\left\{j_{1}, \cdots j_{6}\right\}} \sum_{\left\{-j_{k} \leq m_{k} \leq j_{k}\right\}}(-1)^{\Sigma \sum_{k=1}^{6} j_{k}}\left\{\begin{array}{lll}
j_{1} & j_{2} & j_{3} \\
j_{4} & j_{5} & j_{6}
\end{array}\right\}_{q}
\end{aligned}
$$

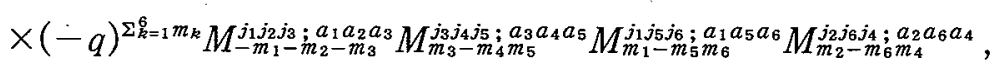

where

$$
\left\{\begin{array}{lll}
j_{1} & j_{2} & j_{3} \\
j_{4} & j_{5} & j_{6}
\end{array}\right\}_{q}
$$

is the Racah-Wigner $6 j$-symbol of the quantum group $S U_{q}(2)^{29)}$ with $q=e^{2 \pi i /(k+2)}$ and

$$
\Lambda_{q}=-\frac{2(k+2)}{\left(q^{1 / 2}-q^{-1 / 2}\right)^{2}}
$$

The integration variable $M_{m_{1} m_{2} m_{3}}^{j_{1} j_{2} j_{3} a_{1} a_{2} a_{3}}$ obeys the conditions

(1) $M_{m_{1} m_{2} m_{3}}^{j_{1} j_{2} j_{3} ; a_{1} a_{2} a_{3}}=0$ unless $\left|j_{1}-j_{2}\right| \leq j_{3} \leq j_{1}+j_{2}$ and $j_{1}+j_{2}+j_{3} \leq k$,

(2) $\dot{\bar{M}}_{m_{1} m_{2} m_{3}}^{j_{1} j_{2} j_{3} a_{1} a_{2} a_{3}}=(-1)^{j_{1+j^{2}+j^{3}}}(-q)^{m_{1}+m_{2}+m_{3}} M_{-m_{1}-m_{2} m_{3}}^{j_{1} j_{2} j_{3} ; a_{1} a_{2} a_{3}}$,

(3) $M_{m_{1} m_{2} m_{3}}^{j_{1} j_{2} j_{3} a_{1} a_{1} a_{2} a_{3}}=M_{m_{2} m_{3} m_{1}}^{j_{2} j_{3} j_{1} ; a_{2} a_{3} a_{1}}$.

The perturbative expansion of this integral gives a sum over Feynman graphs each of which is dual to an oriented simplicial complex in three dimensions. A contribution of each simplicial complex $T$ is evaluated by assigning a triplet of indices $\left(a_{e}, j_{e}, m_{e}\right)$ to each edge $e$ in $T$ and by summing over the indices on all the edges with the conditions

$$
\left|j_{e_{1}(\Delta)-} j_{e_{2}(\Delta)}\right| \leq j_{e_{3}(\Delta)} \leq j_{e_{1}(\Delta)}+j_{e_{2}(\Delta)}, \quad j_{e_{1}(\Delta)}+j_{e_{2}(\Delta)}+j_{e_{3}(\Delta)} \leq k
$$

for every triangle $\Delta$ in $T$ where $e_{r}(\Delta)(r=1,2,3)$ are the edges of $\Delta$. We then obtain

$$
\log Z_{q}=\sum_{T} \frac{1}{C(T)}\left(g \Lambda_{q}\right)^{n_{3}(T)} N^{n_{1}(T)} \Lambda_{q}^{n_{0}(T)-1} I_{q}(T)
$$

where the sum $\Sigma_{T}$ is over connected simplicial complexes, and $I_{q}(T)$ is given by 


$$
\begin{aligned}
I_{q}(T)= & \frac{1}{\Lambda_{p}^{n_{0}(T)-1}} \sum_{\{j\}_{e: \text { edges }}}(-1)^{2 j_{e}}\left[2 j_{e}+1\right]_{q} \\
& \times \prod_{t: \text { tetrahedra }}(-1)^{\Sigma \xi_{k-1}^{\xi} j_{e k}(t)}\left\{\begin{array}{lll}
j_{e_{1}(t)} & j_{e_{2}(t)} & j_{e_{3}(t)} \\
j_{e_{4}(t)} & j_{e_{5}(t)} & j_{e_{6}(t)}
\end{array}\right\}_{q},
\end{aligned}
$$

where

$$
[2 j+1]_{q}=\frac{q^{j+(1 / 2)}-q^{-j-(1 / 2)}}{q^{1 / 2}-q^{-1 / 2}},
$$

and $e_{r}(t)(r=1, \cdots, 6)$ are the six edges of the tetrahedron $t$.

It has been shown by Turaev and Viro ${ }^{26)}$ that $I_{q}(T)$ is a topological invariant of $T$, i.e., $\quad I_{q}\left(T_{1}\right)=I_{q}\left(T_{2}\right)$ if $T_{1}$ and $T_{2}$ are combinatorially equivalent. Especially when $T$ is a triangulation of an orientable manifold $M$, it has been shown in Refs. 30) and 31) that $I_{q}(T)$ is equal to $\left|\tau_{q}(M)\right|^{2}$ where $\tau_{q}(M)$ is the Witten-Reshetikhin-Turaev invariant. $^{32)}$ (The relation between $I_{q}(T)$ and $\tau_{q}(M)$ at $q \rightarrow 1$ has also been studied in 33) and 34). See also Refs. 35) and 36).) For example,

$$
I_{q}\left(S^{3}\right)=1, \quad I_{q}\left(S^{1} \times S^{2}\right)=\Lambda_{q} .
$$

In general, it has been shown by $\mathrm{Kohno}^{37)}$ that $I_{q}(M)$ is bounded from above as

$$
I_{q}(M) \leq \Lambda_{q}^{h(M)}
$$

where $h(M)$ is the Heegaard genus. Any three-dimensional manifold can be decomposed into a pair of handlebodies $\mathscr{H}_{h}$ of genus $h$ glued together on their boundaries (the Heegaard splitting), and the Heegaard genus $h(M)$ is the minimum number of such $h$. Especially $h(M)=0$ if and only if $M$ is homeomorphic to $S^{3}$ and otherwise $h(M) \geq 1$.

By introducing a new variable $\mu$ by $N=\mu / \Lambda_{q}$, the partition function $Z_{q}$ is expressed as

$$
\log Z_{q}=\frac{1}{\Lambda_{q}} \sum_{T} \frac{1}{C(T)} g^{n_{3}(T)} \mu^{n_{1}(T)} \Lambda_{q}^{\chi(T)} I_{q}(T)
$$

Suppose that we could take the limit $q \rightarrow 0$ so that $\Lambda_{q} \rightarrow 0$ while maintaining the inequality (14). As we mentioned in the introduction, $\chi(T) \geq 0$ for any simplicial complex $T$ in three dimensions and the equality holds if and only if $T$ is a simplicial manifold. Because of the inequality (14), the $q \rightarrow 0$ limit of $\log Z_{q}$ would be dominated by a sum over simplicial manifolds with $\chi(T)=0$ and $h(T)=0$, i.e., $T \simeq S^{3}$. Unfortunately, it is not clear how to make sense of this limit since the model is defined only when $q$ is a root of unity. In the following, we will develop as alternative mechanism to impose the condition $T \simeq S^{3}$ without using the $q \rightarrow 0$ limit.

\section{§. Schwinger-Dyson equation in three dimensions}

In the case of the matrix model, the integral over the hermitian matrix $M$ in (1) can be reduced to an integral over the eigenvalues $\lambda_{i}(i=1, \cdots, N)$ of $M$ 


$$
\int[d M] \exp (-S(M))=\int \prod_{i=1}^{N} d \lambda_{i} \prod_{i<j}\left(\lambda_{i}-\lambda_{j}\right)^{2} \exp \left(-\sum_{i}\left(\frac{1}{2} \lambda_{i}{ }^{2}-\frac{g}{3 \sqrt{N}} \lambda_{i}{ }^{3}\right)\right),
$$

and the powerful methods of the WKB approximation ${ }^{4)}$ and the orthogonal polynomials $^{8)}$ have been used to successfully analyze the model. This approach, however, does not seem to have an obvious generalization to three dimensions. It is not clear if the integral over $M_{m_{1} m_{2} m_{3}}^{j_{1} j_{2} j_{3} a_{2} a_{1} a_{2} a_{3}}$ could be significantly reduced by introducing some variables similar to the eigenvalues.

In $\S 2$, we saw that the large- $N$ matrix model can also be studied by using the Schwinger-Dyson equation for $u_{n}$, the expectation value of $\operatorname{tr} M^{n}$. In fact $u_{n}$ is the integral transform (9) of $\rho(\lambda)$, the density of the eigenvalue. This is not too surprising since both the WKB approximation and the Schwinger-Dyson equation at large$N$ make use of the classical equation of motion of the model. The point here is that the method of the Schwinger-Dyson equation is applicable to the three-dimensional case.

In the case of the matrix model, the expectation value of $\operatorname{tr} M^{n}$ is expressed as a sum over planar graphs with $n$ external legs. Thus $\operatorname{tr} M^{n}$ may be regarded as an operator which creates a circle with $n$ edges in each of the planar graphs. The natural thing to consider in three dimensions should then be an operator which creates a closed connected triangulated surface $\Sigma$. Indeed we can construct such an operator as follows. We attach a triplet of indices $\left(j_{e}, m_{e}, a_{e}\right)$ to each edge $e$ on the surface $\Sigma$ and associate $M_{m_{1} m_{2} m_{3}}^{j_{1} j_{2} j_{3} a_{1} a_{2} a_{2}}\left(j_{r}=j_{e r(\Delta)}, m_{r}= \pm m_{e_{r}(\Delta),}, a_{r}=a_{e_{r}(\Delta)}\right)$ to each triangle $\Delta$. The signs $( \pm)$ of $m_{r}$ for a pair of triangle sharing the edge $e$ are chosen to be opposite. We then define a polynomial $F_{\Sigma}(j ; M)$ of $M$ by summing over $\{M\}$ and $\{a\}$ as

$$
\begin{aligned}
& F_{\Sigma}(j ; M)=\sum_{\{m\},\left\{a_{e ; ; \text { dgges }}\right.} \frac{(-1)^{j_{e}}}{2}\left[(-q)^{m_{e}}+(-q)^{-m_{e}}\right]
\end{aligned}
$$

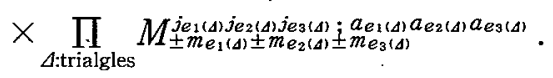

By taking an expectation value of $F_{\Sigma}$, we obtain a function $\Psi_{\Sigma}(j)$ of the indices $\{j\}$ on $\Sigma$ as

$$
\Psi_{\Sigma}(j)=\left\langle F_{\Sigma}(j ; M)\right\rangle=\frac{1}{Z} \int[d M] F_{\Sigma}(j ; M) \exp (-S(M))
$$

We have not yet summed over the indices $\{j\}$.

We saw in Eq. (13) that the weight $I_{q}(T)$ for the simplicial complex $T$ in the summation (15) is given by a sum over $\{j\}$ on the edges of $T$. We may regard $I_{q}(T)$ as a partition function of a lattice statistical model on $T$. In such a topological lattice model, we can introduce a notion of physical states on the closed triangulated surface $\Sigma$ as follows. ${ }^{26,33) 34)}$ We start with a space $V_{\Sigma}$ spanned by functions $\Phi(j)$ of $\{j\}$ on $\Sigma$ and call it a space of states. In order to define physical states in $V_{\Sigma}$, we consider a three-dimensional simplicial complex $T_{\Sigma_{0}, \Sigma_{1}}$ which is homeomorphic to $\Sigma \times[0,1]$ and whose boundaries $\Sigma \times\{0\}$ and $\Sigma \times\{1\}$ are triangulated as $\Sigma_{0}$ and $\Sigma_{1}$. We then define a map $P_{\Sigma_{0}, \Sigma_{1}}$ from $V_{\Sigma_{0}}$ to $V_{\Sigma_{1}}$ by 


$$
P_{\Sigma_{0}, \Sigma_{1}:} \Phi(j) \in V_{\Sigma_{0}} \rightarrow \sum_{\left\{j^{\prime}\right\}} \Phi\left(j^{\prime}\right) P_{\Sigma_{0}, \Sigma_{1}}\left(j^{\prime}, j\right) \in V_{\Sigma_{1}}, \quad .
$$

where $P_{\Sigma_{0}, \Sigma_{1}}\left(j^{\prime}, j\right)$ is defined by attaching an index $J_{e}$ on each edge $e$ on $T_{\Sigma_{0}, \Sigma_{1}}$ and by summing over $\{J\}$ as

$$
\begin{aligned}
& P_{\Sigma_{0}, \Sigma_{1}}\left(j^{\prime}, j\right)=\frac{\Lambda_{q}^{n_{0}\left(\Sigma_{1}\right)}}{\Lambda_{q}^{n_{0}\left(T_{\left.\Sigma 0, \Sigma_{1}\right)}\right)}} \sum_{\{J\}} \prod_{e \in \Sigma \times\{0\}} \delta\left(J_{e}, j_{e}\right) \\
& \times \prod_{e \in T_{\Sigma} \times\{1\}}\left(\frac{\delta\left(J_{e}, j_{e}^{\prime}\right)}{(-1)^{2 j_{j}^{\prime}}\left[2 j_{e}^{\prime}+1\right]_{q}}\right)_{e \in T_{T_{S_{0}, \Sigma_{1}}}}(-1)^{2 J_{e}}\left[2 J_{e}+1\right]_{q}
\end{aligned}
$$

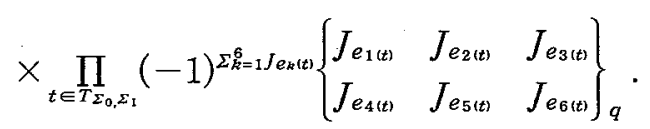

Because of the topological invariance, $P_{\Sigma_{0}, \Sigma_{1}}$ is independent of the choice of the triangulation in the interior of $T_{\Sigma_{0}, \Sigma_{1}}$ and satisfies ${ }^{26)}$

$$
\sum_{\left\{j^{\prime}\right\}} P_{\Sigma_{0}, \Sigma_{1}}\left(j, j^{\prime}\right) P_{\Sigma_{1}, \Sigma_{2}}\left(j^{\prime}, j^{\prime \prime}\right)=P_{\Sigma_{0}, \Sigma_{2}}\left(j, j^{\prime \prime}\right),
$$

where $\Sigma_{0}, \Sigma_{1}$ and $\Sigma_{2}$ are all homeomorphic to $\Sigma$. Especially when $\Sigma_{0}=\Sigma_{1}=\Sigma, P_{\Sigma, \Sigma}$ acts as a projection operator on $V_{\Sigma}$. The physical subspace $V_{\Sigma}^{\text {(phys) }}$ is defined as a subspace of $V_{\Sigma}$ projected out by $P_{\Sigma, \Sigma}$ as

$$
V_{\Sigma}^{\text {(phys) }}=\left\{\Phi \in V_{\Sigma}: \sum_{\left\{j^{\prime}\right\}} \Phi\left(j^{\prime}\right) P_{\Sigma, \Sigma}\left(j^{\prime}, j\right)=\Phi(j)\right\} .
$$

Because of the property (19), the operator $P_{\Sigma_{0}, \Sigma_{1}}$ defines an invertible map from a physical state in $V_{\Sigma_{1}}$ onto a physical state in $V_{\Sigma_{0}}$. Therefore $V_{\Sigma_{0}}^{\text {(phys) }}$ and $V_{\Sigma_{1}}^{\text {(phys) }}$ are isomorphic.

Since $\Psi_{\Sigma}(j)$ given by (17) is also a function of $\{j\}$, we may consider its pairing with a physical state $\Phi$ on $\Sigma$ as

$$
\left(\Phi, \Psi_{\Sigma}\right)=\Lambda_{q}^{(1 / 2) n_{2}(\Sigma)+h(\Sigma)} \sum_{\{j\}} \Phi(j) \Psi_{\Sigma}(j)
$$

where $h(\Sigma)$ is the genus of $\Sigma$. The factor $\Lambda_{q}^{(1 / 2) n_{2}(\Sigma)+h(\Sigma)}$ will simplify the equations in the following. As we shall see below, $\Phi_{\Sigma}(j)$ itself is not a physical state, and the pairing $\left(\Phi, \Psi_{\Sigma}\right)$ depends on the triangulation $\Sigma$ of the surface. The Schwinger-Dyson equation describes the dependence of $\left(\Phi, \Psi_{\Sigma}\right)$ on $\Sigma$.

Among physical states on $\Sigma$, especially important are the Hartle-Hawking type states $^{34)}$ defined as follows. A handlebody $\mathscr{H}_{h}$ of genus $h$ is obtained by cutting out $2 h$ pairs of discs from a boundary of a three-dimensional ball $B^{3}$ and by identifying them pairwisely in opposite orientations. The boundary of $\mathscr{H}_{h}$ is then a closed orientable surface of genus $h$. The boundaries of the discs become simple homology cycles on the boundary of $\mathscr{H}_{h}$ which do not intersect with each other and which are contractible in $\mathscr{H}_{h}$. They are called the meridians of the handlebody. Let us consider a simplicial manifold $\mathcal{H}_{\Sigma}^{(\alpha)}$ homeomorphic to a handlebody of genus $h$ whose boundary is triangulated as on $\Sigma(h(\Sigma)=h)$ and whose meridians are given by $\left\{\alpha_{1}, \cdots\right.$, $\left.\alpha_{h}\right\}$ where each $\alpha_{i}$ is chosen to be a sequence of edges on $\Sigma$. The Hartle-Hawking type state $\Phi^{(a)}(j)$ associated to the handlebody is defined by 


$$
\begin{aligned}
& \Phi^{(\alpha)}(j)=\frac{\Lambda_{q}^{n_{0}(\Sigma)}}{\left.\Lambda_{q}^{n_{0}\left(H_{2}(\alpha)\right.}\right)} \sum_{\{j\}} \prod_{e \in \Sigma}\left(\frac{\delta\left(J_{e}, j_{e}\right)}{(-1)^{2 j e}\left[2 j_{e}+1\right]_{q}}\right) \prod_{e \in \mathscr{H}_{\Sigma}^{(\alpha)}}(-1)^{2 J e}\left[2 J_{e}+1\right]_{q} \\
& \times \prod_{t \in \mathscr{S}((\alpha))}(-1)^{\sum_{h=1}^{\delta} J_{e_{n}(t)}}\left\{\begin{array}{lll}
J_{e_{1}(t)} & J_{e_{2}(t)} & J_{e_{3}(t)} \\
J_{e_{4}(t)} & J_{e_{5}(t)} & J_{e_{6}(t)}
\end{array}\right\}_{q} .
\end{aligned}
$$

Because of the topological invariance, $\Phi^{(\alpha)}(j)$ defined in this way is independent of the choice of the triangulation of the interior of the handlebody, and it gives a physical state on $\Sigma$.

By expanding integral (17) in powers of $g$, the pairing of $\Psi_{\Sigma}$ with the HartleHawking type state $\Phi^{(\alpha)}$ is expressed as

$$
\begin{aligned}
\left(\Phi^{(\alpha)}, \Psi_{\Sigma}\right)= & \Lambda_{q}^{(1 / 2) n_{2}(\Sigma)+h(\Sigma)} \sum_{\{j\}} \Phi^{(\alpha)}(j)\langle F(j ; M)\rangle \\
= & \Lambda_{q}^{(1 / 2) n_{2}(\Sigma)+h(\Sigma)} \sum_{T: \partial T=\Sigma}\left(\frac{C(\Sigma)}{C(T)}\right)\left(g \Lambda_{q}\right)^{n_{3}(T)}\left(\frac{\mu}{\Lambda_{q}}\right)^{n_{1}(T)} \\
& \times \Lambda_{q}^{n_{0}(T)-1} I_{q}\left(\mathcal{H}_{\Sigma}^{(\alpha)} \cup_{\Sigma}(-T)\right) \\
= & \sum_{T: \partial T=\Sigma}\left(\frac{C(\Sigma)}{C(T)}\right) g^{n_{3}(T)} \mu^{n_{1}(T)} \Lambda_{q}^{\chi(T)-(1 / 2) \chi(\Sigma)} I_{q}\left(\mathcal{H}_{\Sigma}^{(\alpha)} \bigcup_{\Sigma}(-T)\right) ;
\end{aligned}
$$

where the sum $\Sigma_{r}$ is over simplicial complexes whose boundary is $\Sigma$ and $I_{q}\left(\mathscr{H}_{\Sigma}^{(\alpha)}\right.$ $\times \cup_{\Sigma}(-T)$ ) is the Turaev-Viro invariant (13) for the simplicial complex obtained by gluing $\mathcal{H}_{\Sigma}^{(\alpha)}$ and $T$ together on their boundaries after inverting the orientation of $T$. As in the case of the matrix model, the dual graph of $T$ is not necessarily connected though it does not contain a closed disconnected part. Especially a triangle on the boundary $\Sigma$ of $T$ may be attached to another triangle on $\Sigma$. For any simplicial complex $T$ bounded by $\Sigma$, its Euler number $\chi(T)$ is greater than or equal to $(1 / 2) \chi(\Sigma)$, and the equality holds if and only if $\mathscr{G}_{\Sigma}^{(\alpha)} \cup(-T)$ is a simplicial manifold. If we could take the limit $q \rightarrow 0$, the right-hand side in the above would become a sum over $T$ such that $\chi(T)=(1 / 2) \chi(\Sigma)$ and $\mathscr{H}_{\Sigma}^{(\alpha)} \cup_{\Sigma}(-T) \simeq S^{3}$. Although this limit itself is not well-defined, we will find a set of factorization conditions which together with the Schwinger-Dyson equation imposes similar conditions on $T$.

In order to derive the Schwinger-Dyson equation, we choose a triangle $\Delta_{0}$ on $\Sigma$ and consider an open surface $\Sigma_{\Delta_{0}}$ obtained by removing the triangle $\Delta_{0}$ from $\Sigma$. Associated to $\Sigma_{\Delta 0}$, we defined a boundary operator $F_{\Sigma_{\Delta 0}}(j ; M)_{m_{1} m_{2} m_{3}}^{a_{1} a_{2} a_{3}}$ by

$$
\begin{aligned}
& F_{\Sigma}(j ; M)_{m_{1} m_{2} m_{3}}^{a_{1} a_{2} a_{3}}=\sum_{\{m\},\{a\}} \prod_{r=1}^{3} \delta\left(m_{r}, m_{e r}\left(\Delta_{0}\right)\right) \delta\left(a_{r}, a_{e r\left(\Delta \Delta_{0}\right)}\right) \\
& \times \prod_{e: \text { edges }} \frac{(-1)^{j e}}{2}\left[(-q)^{m_{e}}+(-q)^{-m_{e}}\right]
\end{aligned}
$$

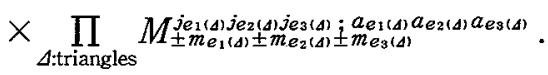

As in the case of the matrix model, we make use of the identity 


$$
\frac{1}{Z} \int[d M] \sum_{\{m\}_{s}\{a\}} \frac{\partial}{\partial M_{m_{1} m_{2} m_{3}}^{j_{j} j_{3} a_{1} a_{2} a_{2} a_{3}}}\left[F_{\Sigma_{\Delta_{0}}}(j ; M)_{m_{1} m_{2} m_{3}}^{a_{1} a_{2} a_{3}} \exp (-S(M))\right]=0,
$$

where we have set $j_{e\left(\Delta_{0}\right)}=j_{r}(r=1,2,3)$. By performing the derivative with respect to $M$, we obtain

$$
\begin{aligned}
\Psi_{\Sigma}(j)= & g \Lambda_{q} \sum_{j_{4}, j j_{5}, j_{6}}(-1)^{\Sigma_{k=1}^{b} j_{k}}\left\{\begin{array}{ccc}
j_{1} & j_{2} & j_{3} \\
j_{4} & j_{5} & j_{6}
\end{array}\right\} \Psi_{\Sigma_{d_{0}}}(j) \\
& +\sum_{\{m\},\{a\}}\left\langle\frac{\partial F_{\Sigma_{d_{0}}}(j ; M)_{m_{1} m 2 m_{3}}^{a_{1} a_{2} a_{3}}}{\partial M_{m_{1} m_{2} m_{3}}^{j_{1} j_{2} j_{3} a_{1} a_{2} a_{3}}}\right\rangle,
\end{aligned}
$$

where $\Sigma_{\tilde{\Delta}_{0}}$ is a triangulated surface obtained from $\Sigma$ by decomposing $\Delta_{0}$ into three small triangles, and $j_{4}, j_{5}, j_{6}$ are spins on the three additional edges on $\Sigma_{\tilde{\Delta}_{0}}$. If it were not for the last term on the right-hand side of Eq. (23), $\Psi_{\Sigma}$ would transform like a physical state under the change of triangulation $\Sigma \rightarrow \Sigma_{\tilde{\Delta}_{0}}$.

Since each $M$ in $F_{\Sigma_{\Delta_{0}}}(j ; M)$ corresponds to a triangle $\Delta$ on $\Sigma_{\Delta_{0}}$, the last term $\left\langle(\partial / \partial M) F_{\Sigma_{\Delta_{0}}}(j ; M)\right\rangle$ on the right-hand side of (23) can be expressed as a sum over closed surfaces $\Sigma_{\Delta, \Delta_{0}}$ obtained by removing $\Delta$ and $\Delta_{0}$ from $\Sigma$ and by identifying their edges. Let us denote the number of common edges of the two triangles by $E\left(\Delta, \Delta_{0}\right)$ and the number of common vertices by $V\left(\Delta, \Delta_{0}\right)$. We can classify the position of $\Delta$ on $\Sigma_{\Delta_{0}}$ according to the values of $E$ and $V$ as follows.

(1) When $E\left(\Delta, \Delta_{0}\right) \neq 0$ and $V\left(\Delta, \Delta_{0}\right)=E\left(\Delta, \Delta_{0}\right)+1, \Delta$ is in the neighborhood of $\Delta_{0}$ on $\Sigma$. In this case $\Sigma_{\Delta, \Delta_{0}}$ has the same topology as $\Sigma$.

(2) When $E\left(\Delta, \Delta_{0}\right)=0$ and $V\left(\Delta, \Delta_{0}\right)=0,1$, the removal of the triangles will create a new handle on $\Sigma$ and the edges of $\Delta_{0}$ becomes a homology cycle around the handle.

(3) When $V\left(\Delta, \Delta_{0}\right) \geq E\left(\Delta, \Delta_{0}\right)+2$, the triangles $\Delta$ and $\Delta_{0}$ wrap around a cycle on $\Sigma$, and $\Sigma_{\Delta, \Delta_{0}}$ is degenerate around the cycle.

Let us consider a generic situation when $E\left(\Delta, \Delta_{0}\right)+1 \geq V\left(\Delta, \Delta_{0}\right)$ for any triangle $\Delta$ on $\Sigma_{\Delta_{0}}$, which includes cases (1) and (2). We also assume here that the surface $\Sigma$ is not degenerate in the neighborhood of $\Delta_{0}$. The cases involving degenerate surfaces will be examined in the next section.

In the generic situation, by multiplying an arbitrary physical state $\Phi$ on both sides of Eq. (23) and by summing over $\{j\}$, we obtain

$$
\begin{aligned}
\left(\Phi, \Psi_{\Sigma}\right)=g\left(\Phi, \Psi_{\Sigma_{\bar{\Delta}_{0}}}\right) & +\sum_{\Delta: E\left(\Delta, \Delta_{0}\right) \neq 0} \mu^{E\left(\Delta, \Delta_{0}\right)}\left(\Phi, \Psi_{\Sigma_{\Delta, \Delta_{0}}}\right) \\
& +\sum_{\Delta: E\left(\Delta, \Delta_{0}\right)=0}\left(i[\Phi]_{\Delta, \Delta_{0}}, \Psi_{\Sigma_{\Delta, \Delta_{0}}}\right)
\end{aligned}
$$

The second term on the right-hand side is a sum over $\Delta$ in the neighborhood of $\Delta_{0}$, and $\Sigma_{\Delta, \Delta_{0}}$ is of the same topology as $\Sigma$. On the other hand, $h\left(\Sigma_{\Delta, \Delta_{0}}\right)=h(\Sigma)+1$ in the last term, and $i_{\Delta, \Delta_{0}}$ is defined by 


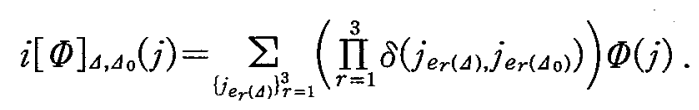

It is easy to show that this gives a map from $V_{\Sigma}^{\text {(phys) }}$ into $V_{\Sigma_{s, \Delta} \Delta_{0}}^{\text {(phys) }}$.

Especially when $\Phi$ is the Hartle-Hawking type state $\Phi^{(\alpha)}, i\left[\Phi^{(\alpha)}\right]_{\Delta, \Delta_{0}}$ is also the Hartle-Hawking type state associated to a handlebody whose boundary in $\Sigma_{\Delta, \Delta_{0}}$ and whose meridians are $\left\{\alpha_{1}, \cdots, \alpha_{i(\Sigma)}, \partial \Delta_{0}\right\}$

$$
i\left[\Phi^{(\alpha)}\right]_{\Delta, \Delta_{0}}(j)=\Phi^{\left(\alpha \cup\left\{\partial \Delta_{0}\right\}\right)}(j),
$$

where $\partial \Delta_{0}$ is the boundary of $\Delta_{0}$. Equation (24) for $\Phi=\Phi^{(\alpha)}$ can then be written as

$$
\left\langle\Sigma^{(\alpha)}\right\rangle=g\left\langle\Sigma_{\beth_{0}}^{(\alpha)}\right\rangle+\sum_{\Delta: E\left(\Delta, \Delta_{0}\right) \neq 0} \mu^{E\left(\Delta, \Delta_{0}\right)}\left\langle\Sigma_{d, \Delta_{0}}^{(\alpha)}\right\rangle+\sum_{\Delta: E\left(\Delta ; \Delta_{0}\right)=0}\left\langle\sum_{\Delta, \Delta_{0}}^{\left(\alpha,\left\{\partial \Delta_{0}\right\}\right)}\right\rangle,
$$

where

$$
\left\langle\Sigma^{(\alpha)}\right\rangle=\left(\Phi^{(\alpha)}, \Psi_{\Sigma}\right)=\Lambda_{q}^{(1 / 2) n_{2}(\Sigma)+h(\Sigma)} \sum_{\{j\}} \Phi^{(\alpha)}(j)\left\langle F_{\Sigma}(j ; M)\right\rangle .
$$

We call this the Schwinger-Dyson equation for the amplitude $\left\langle\Sigma^{(\alpha)}\right\rangle$ of the triangulated surface $\Sigma$ with the choice $\left\{\alpha_{i}\right\}$ of simple homology cycles. We see that, when $\Sigma_{\Delta, \Delta_{0}}$ is non-degenerate, the Schwinger-Dyson equation (26) does not depend on the value of $q$ explicitly.

\section{$\S 5$. Restriction on topologies}

\subsection{General case}

We have found that the Schwinger-Dyson equation (26) takes the same form for any value of $q$ as far as the surface $\Sigma$ in the equation is non-degenerate. On the other hand, the amplitude $\left\langle\Sigma^{(\alpha)}\right\rangle$ which solves the equation is expanded as a sum over $T$ weighted with $\Lambda_{q}^{\chi(T)-(1 / 2) \chi(\Sigma)} I_{q}\left(\mathcal{H}_{\Sigma}^{(\alpha)} U_{\Sigma}(-T)\right)$ as in Eq. (22). Depending on the topology of $T$, the weight takes various functional forms in $q$. Since the Schwinger Dyson equation for non-degenerate surfaces is linear in $\left\langle\Sigma^{(\alpha)}\right\rangle$, a sum over a restricted class of triangulations $T$ such that the weight takes the same functional form should also give a solution to the equation.

Here we claim a stronger statement that, for each choice of a compact orientable three-dimensional manifold $M$, a sum over triangulations $T$ such that

$$
\mathscr{H}_{\Sigma}^{(\alpha)} \cup_{\Sigma}(-T) \simeq M
$$

satisfies the Schwinger-Dyson equation. Being a triangulation of the manifold, $T$ should also satisfy $\chi(T)=(1 / 2) \chi(\Sigma)$. Let us define

$$
\begin{aligned}
& \left\langle\Sigma^{(\alpha)}\right\rangle_{\mid M}=\sum_{T: \mathscr{H}_{r}^{(a)} \sum_{U_{\tau}(-T) \approx M}}\left(\frac{C(\Sigma)}{C(T)}\right) g^{n_{3}(T)} \mu^{n_{1}(T)} \\
& =\sum_{n_{3}, n_{1}} Z_{n_{3}, n_{1}}(\Sigma ; \alpha)_{\mid M} g^{n_{3}} \mu^{n_{1}},
\end{aligned}
$$


where the sum $\Sigma_{T}$ is over triangulations $T$ satisfying the condition (28), and $Z_{n_{3}, n_{1}}$ $(\Sigma ; \alpha)_{\mid M}$ is the number of such triangulations with $n_{3}(T)=n_{3}, n_{1}(T)=n_{1}$. We are going to show that this gives a solution to the Schwinger-Dyson equation.

By definition, for each triangulation $T$ counted in the above, $\mathcal{H}_{\Sigma}^{(a)} \cup_{\Sigma}(-T)$ give a triangulation of $M$. Let us pay attention to one of the triangles $\Delta_{0}$ on the boundary $\Sigma$ of $T$. In the simplicial complex $T$, the triangle $\Delta_{0}$ may be either attached to a tetrahedron or identified with another triangle $\Delta$ on $\Sigma_{\Delta_{0}}$.

If $\Delta_{0}$ is attached to a tetrahedron in $T$, we can remove the tetrahedron from $T$ and give it to the handlebody $\mathcal{H}_{\Sigma}^{(\alpha)}$, without changing the triangulation of $M$. After removing the tetrahedron, $T$ becomes a triangulation $T^{\prime}$ consisting of $\left(n_{3}-1\right)$ tetrahedron whose boundary is $\Sigma_{\bar{\Delta}_{0}}$. Obviously $T^{\prime}$ obeys $\chi\left(T^{\prime}\right)=(1 / 2) \chi\left(\Sigma_{\vec{\Delta}_{0}}\right)$ if $\chi(T)$ $=(1 / 2) \chi(\Sigma)$. The handlebody $\mathscr{H}_{\Sigma}^{(\alpha)}$ becomes $\mathscr{H}_{\Sigma_{\Sigma_{0}}}$ after adding the tetrahedron on it. Since the triangulation of $M$ remains unchanged, $T^{\prime}$ should satisfy

$$
\mathscr{H}_{\Sigma_{\overline{\mathcal{J}}_{0}}}{ }^{(\alpha)} \cup_{\Sigma_{\overline{\mathcal{A}}_{0}}}\left(-T^{\prime}\right) \simeq M
$$

Such $T^{\prime}$ is counted in $Z_{n_{3}-1, n_{1}}\left(\Sigma_{\widehat{\Delta}_{0}} ; \alpha\right)_{\mid M}$. Conversely, from any triangulation $T^{\prime}$ satisfying the above, we can construct a triangulation $T$ such that the condition (28) holds. However the correspondence between $T$ and $T^{\prime}$ is not necessarily one to one when $T$ and $T^{\prime}$ have nontrivial symmetries. We shall see below that this is taken care of by the factors $C(\Sigma) / C(T)$ and $C\left(\Sigma_{\tilde{\Delta}_{0}}\right) / C\left(T^{\prime}\right)$.

Let $G(\Sigma)$ be the symmetry of $\Sigma$ under interchange of the triangles on it, and $G\left(\Sigma_{\Delta_{0}}\right)$ be the invariant subgroup of $G(\Sigma)$ which keeps the position of the triangle $\Delta_{0}$. fixed. Then $\left[G(\Sigma): G\left(\Sigma_{\Delta_{0}}\right)\right]=C(\Sigma) / C\left(\Sigma_{\Delta_{0}}\right)$ gives the number of the triangles on $\Sigma$ which are conjugate to $\Delta_{0}$ under the action of $G(\Sigma)$. Suppose that $m_{1}(T)$ of them are attached to tetrahedra in the interior of $T$, and $m_{2}(T)$ of them are attached to triangles on $\Sigma_{\Delta_{0}}$ so that $m_{1}(T)+m_{2}(T)=C(\Sigma) / C\left(\Sigma_{\Delta_{0}}\right)$. The contribution $C(\Sigma) / C(T)$ of the triangulation $T$ to $Z_{n_{3}, n_{1}}(\Sigma ; \alpha)_{\mid M}$ can then be divided into two parts as

$$
\frac{C(\Sigma)}{C(T)}=C\left(\Sigma_{\Delta_{0}}\right) \cdot\left(\frac{m_{1}(T)}{C(T)}+\frac{m_{2}(T)}{C(T)}\right)
$$

Let us show that the sum

$$
C\left(\Sigma_{\Delta_{0}}\right) \cdot \sum_{T}\left(\frac{m_{1}(T)}{C(T)}\right)
$$

over triangulations $T$ which satisfy Eq. (28) and $n_{3}(T)=n_{3}, n_{1}(T)=n_{1}$ is equal to $Z_{n_{3}-1, n_{1}}\left(\Sigma_{\bar{\Delta}_{0}} ; \alpha\right)_{1 M}$. If we remove a tetrahedron attached to one of the $m_{1}(T)$ triangles conjugate to $\Delta_{0}$, we obtain a triangulation $T^{\prime}$ consisting of $\left(n_{3}-1\right)$ tetrahedra. It may happen that there is more than one way to remove a tetrahedron from $T$ to obtain the same triangulation $T^{\prime}$. Let $N\left(T \rightarrow T^{\prime}\right)$ be the number of ways to obtain $T^{\prime}$ from $T$. Obviously $m_{1}(T)=\Sigma_{T^{\prime}} N\left(T \rightarrow T^{\prime}\right)$, and the summation given by Eq. (31) is equal to

$$
C\left(\Sigma_{\Delta_{0}}\right) \cdot \sum_{T} \sum_{T^{\prime}}\left(\frac{N\left(T \rightarrow T^{\prime}\right)}{C(T)}\right) .
$$

The number $N\left(T \rightarrow T^{\prime}\right)$ can be expressed as 


$$
N\left(T \rightarrow T^{\prime}\right)=\frac{C(T)}{C\left(T, T^{\prime}\right)},
$$

where $C\left(T, T^{\prime}\right)$ is the order of the symmetry of $T$ which keeps the position of the tetrahedron at $T \backslash T^{\prime}$ fixed. Therefore Eq. (31) can be expressed as

$$
C\left(\Sigma_{\Delta_{0}}\right) \cdot \sum_{T} \sum_{T^{\prime}} \frac{1}{C\left(T, T^{\prime}\right)}
$$

On the other hand,

$$
\begin{aligned}
Z_{n_{3}-1, n_{1}}\left(\Sigma_{\hat{\Delta}_{0}} ; \alpha\right)_{\mid M} & =C\left(\Sigma_{\Delta_{0}}\right) \cdot \sum_{T^{\prime}}\left(\frac{C\left(\Sigma_{\hat{\Delta}_{0}}\right) / C\left(\Sigma_{\Delta_{0}}\right)}{C\left(T^{\prime}\right)}\right) \\
& =C\left(\Sigma_{\Delta_{0}}\right) \cdot \sum_{T^{\prime}} \sum_{T}\left(\frac{N\left(T \leftarrow T^{\prime}\right)}{C\left(T^{\prime}\right)}\right),
\end{aligned}
$$

where $N\left(T \leftarrow T^{\prime}\right)$ is the number of ways to construct $T$ from $T^{\prime}$ by attaching a tetrahedron on the boundary of $T^{\prime}$. The number $N\left(T \leftarrow T^{\prime}\right)$ can also be expressed as

$$
N\left(T^{\prime} \leftarrow T\right)=\frac{C\left(T^{\prime}\right)}{C\left(T, T^{\prime}\right)}
$$

Combining these, we obtain

$$
\begin{aligned}
Z_{n_{3}-1, n_{1}}\left(\Sigma_{\left.\bar{J}_{0} ; \alpha\right)_{\mid M}}\right. & =C\left(\Sigma_{\Delta_{0}}\right) \cdot \sum_{T^{\prime}} \sum_{T} \frac{1}{C\left(T, T^{\prime}\right)} \\
& =C\left(\Sigma_{\Delta_{0}}\right) \cdot \sum_{T}\left(\frac{m_{1}(T)}{C(T)}\right) .
\end{aligned}
$$

Next we consider the case when $\Delta_{0}$ on the boundary of $T$ is identified with another triangle $\Delta$ on $\Sigma_{\Delta_{0}}$. If $\Delta$ is in the neighborhood of $\Delta_{0}$ on $\Sigma$, i.e., $E\left(\Delta, \Delta_{0}\right) \neq 0$ and $V\left(\Delta, \Delta_{0}\right)=E\left(\Delta, \Delta_{0}\right)+1$, we can remove the triangle $\Delta=\Delta_{0}$ from $T$ to obtain $T^{\prime}$ whose boundary is $\Sigma_{\Delta, \Delta_{0}}$. In this case, the topologies of the boundaries are the same, i.e., $\chi(\Sigma)=\chi\left(\Sigma_{\Delta, \Delta_{0}}\right)$. Since $T^{\prime}$ is obtained from $T$ by removing one triangle, $E\left(\Delta, \Delta_{0}\right)$ edges and $\left(V\left(\Delta, \Delta_{0}\right)-2\right)$ vertices, the Euler number of $T^{\prime}$ remains the same as that of $T ; \chi\left(T^{\prime}\right)=\chi(T)-1+E\left(\Delta, \Delta_{0}\right)-\left(V\left(\Delta, \Delta_{0}\right)-2\right)=\chi(T)$. Therefore $\chi(T)=(1 / 2) \chi(\Sigma)$ implies $\chi\left(T^{\prime}\right)=(1 / 2) \chi\left(\Sigma_{\Delta, \Delta_{0}}\right)$. In this case, the identification of the two triangles

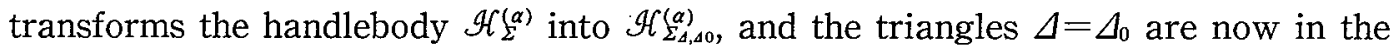
interior of $\mathscr{H}_{\Sigma_{\Delta, \Delta_{0}}}^{(\alpha)}$. By construction, the triangulation $T^{\prime}$ satisfies $\mathcal{H}_{\Sigma_{\Delta, \Delta_{0}}}^{(\alpha)} \cup_{\Sigma_{\Delta \Delta_{0}}}\left(-T^{\prime}\right)$ $\simeq M$.

On the other hand, when $\Delta$ is not in the neighborhood of $\Delta_{0}$, i.e., $E\left(\Delta, \Delta_{0}\right)=0$ and $V\left(\Delta, \Delta_{0}\right)=0,1$, the removal of the triangle $\Delta=\Delta_{0}$ from $T$ creates a hole in $T$. The Euler number of boundary surface then decreases by two, $\chi\left(\Sigma_{\Delta, \Delta_{0}}\right)=\chi(\Sigma)-2$. On the other hand, the numbers of the edges and the vertices of $T^{\prime}$ remain the same as those of $T$. Therefore $\chi\left(T^{\prime}\right)=\chi(T)-1=(1 / 2) \chi\left(\Sigma_{\Delta, \Delta_{0}}\right)$. The handlebody $\mathcal{H}_{\Sigma}^{(\alpha)}$ acquires a new handle after the removal of $\Delta=\Delta_{0}$, and $\Delta_{0}$ becomes a meridian disc of the handle. Therefore $\mathscr{H}_{\Sigma_{d, A_{0}}}^{\left(a \cup\left\{\Delta_{0}\right\}\right)} \cup_{\Sigma_{\Delta, A_{0}}}\left(-T^{\prime}\right) \simeq M$.

As in the case of Eq. (32), we can estimate the contribution of these triangulations 
to $Z_{n_{3}, n_{1}}(\Sigma ; \alpha)_{\mid M}$, taking the symmetry factor $C(\Sigma) / C(T)$ into account, and obtain

$$
\begin{aligned}
C\left(\Sigma_{\Delta_{0}}\right) \cdot \sum_{T}\left(\frac{m_{2}(T)}{C(T)}\right)= & \sum_{\Delta: E\left(\Delta, \Delta_{0}\right) \neq 0} Z_{n_{3}, n_{1}-E\left(\Delta, \Delta_{0}\right)}\left(\Sigma_{\Delta, \Delta_{0} ; \alpha} ;\right)_{\mid M} \\
& +\sum_{\Delta: E\left(\Delta, \Delta_{0}\right)=0} Z_{n_{3}, n_{1}}\left(\Sigma_{\Delta, \Delta_{0} ; \alpha} \cup\left\{\partial \Delta_{0}\right\}\right)_{\mid M} .
\end{aligned}
$$

Combining this with Eq. (32), $Z_{n_{3}, n_{1}}(\Sigma ; \alpha)_{\mid M}$ is expressed as

$$
\begin{aligned}
Z_{n_{3}, n_{1}}(\Sigma ; \alpha)= & Z_{n_{3}-1, n 1}\left(\sum_{\widehat{\Delta}_{0}} ; \alpha\right)+\sum_{\Delta: E\left(\Delta, \Delta_{0}\right) \neq 0} Z_{n_{3}, n_{1}-E\left(\Delta, \Delta_{0}\right)}\left(\sum_{\Delta, \Delta_{0}} ; \alpha\right) \\
& +\sum_{\Delta: E\left(\Delta, \Delta_{0}\right)=0} Z_{n_{3}, n_{1}}\left(\Sigma_{\Delta, \Delta_{0}} ; \alpha \cup\left\{\partial \Delta_{0}\right\}\right) .
\end{aligned}
$$

Therefore $\left\langle\Sigma^{(\alpha)}\right\rangle_{\mid M}$ solves the Schwinger-Dyson equation, for any choice of $M$.

\subsection{Triangulations of handlebodies}

We have seen that the Schwinger-Dyson equation (34) holds for any closed orientable manifold $M$. Especially when $M=S^{3}$, there is a nice characterization of triangulations $T$ counted in $Z_{n_{3}, n_{1}}(\Sigma ; \alpha)_{\left.\right|^{3}}$.

If we remove a handlebody from $S^{3}$, the rest is also a handlebody which is described as follows. Let us first consider the case when $\Sigma$ is isomorphic to a torus. In this case, it is convenient to choose a canonical homology basis $\{\alpha, \beta\}$ such that $\alpha$ is the meridian of the handlebody (solid torus) $\mathscr{I}_{\Sigma}^{(\alpha)}$ and that $\alpha$ and $\beta$ intersect once with each other transversely. The cycle $\beta$ is called the longitude of the handlebody. The choice of the longitude is unique upto $\alpha$, i.e., $\beta+n \alpha$ is also the longitude of $\mathcal{H}_{\Sigma}^{(\alpha)}$. Three-dimensional manifolds with genus-one Heegaard splittings are classified as

$$
\begin{aligned}
& \mathscr{H}_{\Sigma}^{(\alpha)} \cup_{\Sigma}\left(-\mathscr{H}_{\Sigma}^{(n \alpha+\beta)}\right) \simeq S^{3}, \\
& \mathscr{H}_{\Sigma}^{(\alpha)} \cup_{\Sigma}\left(-\mathscr{H}_{\Sigma}^{(n \alpha)}\right) \simeq S^{1} \times S^{2}, \\
& \mathscr{H}_{\Sigma}^{(\alpha)} \cup_{\Sigma}\left(-\mathscr{H}_{\Sigma}^{(n \alpha+m \beta)}\right) \simeq L_{m, n}, \quad(m \neq 0,1) .
\end{aligned}
$$

where $L_{m, n}$ is the lense space. Therefore, if $\mathscr{H}_{\Sigma}^{(\alpha)} \cup_{\Sigma}(-T) \simeq S^{3}, T$ must be a triangulation of a handlebody whose meridian (longitude) is the longitude (meridian) of $\mathscr{H}_{\Sigma}^{(\alpha)}$.

This is also the case for $h(\Sigma) \geq 2$. Corresponding to the meridians $\left\{\alpha_{i}\right\}_{i=1}^{h(\Sigma)}$ of the handlebody $\mathscr{H}_{\Sigma}^{(\alpha)}$, we choose simple homology cycles $\left\{\beta_{i}\right\}$ on $\Sigma$ such that $\alpha_{i}$ and $\beta_{i}(i=1, \cdots, h(\Sigma))$ make a canonical homology basis, i.e., they have the following intersection properties:

$$
\begin{aligned}
& \alpha_{i} \cdot \beta_{j}=\delta_{i j}, \\
& \alpha_{i} \cdot \alpha_{j}=0, \beta_{i} \cdot \beta_{j}=0 .
\end{aligned}
$$

As in the case of torus, the cycles $\left\{\beta_{i}\right\}$ are called the longitudes of $\mathcal{H}_{\Sigma}^{(\alpha)}$. It is known in general that the Heegaard splitting of $S^{3}$ is unique upto the stable equivalence, ${ }^{38)}$ and $T$ satisfying $\mathscr{H}_{\Sigma}^{(\alpha)} \cup_{\Sigma}(-T)$ should be a triangulation of a handlebody whose 
meridians (longitudes) are the longitudes (meridians) of $\mathscr{H}_{\Sigma}^{(\alpha)}$.

The sum over triangulations of the handlebody given by $\left\langle\Sigma^{(\alpha)}\right\rangle_{\mid S^{s}}$ is closely related to the sum over triangulations of $S^{3}$. Before explaining the relation, it is instructive to examine the amplitude $\left\langle\Sigma^{(\alpha)}\right\rangle$ given by Eq. (27) and its relation to $Z_{q}$. Let us consider the case when $\Sigma$ is homeomorphic to $S^{2}$ and is given by a single tetrahedron. For the single tetrahedron, the Hartle-Hawking type state $\Phi$ is given by the $6 j$-symbol and $n_{2}=4$. The boundary operator $\Lambda_{q}^{2} \Sigma_{j} \Phi(j) F(j ; M)$ for the tetrahedron is then equal to the quartic term in the action $S(M)$ upto the factor $(1 / 12) g \Lambda_{q}$. Therefore, as in the case of the matrix model (10), the partition function $Z_{q}$ and the amplitude of the tetrahedron are related as

$$
\begin{aligned}
\langle\text { tetrahedron〉 } & =12 \Lambda_{q} \frac{1}{Z} \frac{\partial Z}{\partial g} \\
& =\sum_{T}\left(\frac{12}{C(T)}\right) n_{3}(T) g^{n_{3}(T)-1} \mu^{n_{1}(T)} \Lambda_{q}^{x(T)} I_{q}(T) .
\end{aligned}
$$

On the other hand, 〈tetrahedron〉 is also expanded as a sum over simplicial complexes $T$ bounded by the tetrahedron as in Eq. (22). Thus the number of closed simplicial complexes $T$ weighted with $\Lambda_{q}{ }^{x(T)} I_{q}(T)$ and the symmetry factor $1 / C(T)$ is equal to $12 n_{3}$ times the number of open simplicial complexes $T$ such that $\partial T=$ (tetrahedron) weighted with $\Lambda_{q}^{\chi(T)-2} I_{q}(($ tetrahedron $) \cup(-T))$ and the symmetry factor $(12 / C(T))$.

A similar relation holds between $\left\langle\Sigma^{(\alpha)}\right\rangle_{\mid S^{3}}$ and the sum over triangulations of $S^{3}$. When $\Sigma$ is a single tetrahedron, the amplitude is a sum over triangulations of a three-dimensional ball $B^{3}$ whose boundary is the tetrahedron.

$$
\left.\langle\text { tetrahedron }\rangle_{\mid S^{3}}=\sum_{n_{3}, n_{1}} Z_{n_{3}, n_{1}} \text { (tetrahedron }\right)_{\mid S^{3}} \cdot g^{n_{3}} \mu^{n_{1}} \text {. }
$$

Here $Z_{n_{3}, n_{1}}$ (tetrahedron) $\left.\right|_{\mid S^{3}}$ is the number of triangulations of the interior of the tetrahedron with $n_{3}$ tetrahedra and $n_{1}$ edges weighted with the symmetry factor $(12 / C(T))$. The factor 12 is the order of the symmetry of the tetrahedron. We can show that this is related to $Z_{n_{3}, n_{1}}\left(S^{3}\right)$ as

$$
Z_{n_{3}-1, n_{1}}(\text { tetrahedron })_{S^{3}}=12 n_{3} Z_{n_{3}, n_{1}}\left(S^{3}\right) \text {. }
$$

Any triangulation of $B^{3}$ is obtained by removing a tetrahedron from a triangulation of $S^{3}$, and conversely any triangulation of $S^{3}$ is given by gluing a tetrahedron to a triangulation of $B^{3}$. For each triangulation $T$ of $S^{3}$, there are $n_{3}(T)$ distinct triangulations of $B^{3}$ as far as $T$ does not have a symmetry. On the other hand, if $T$ has a non-trivial symmetry, different choices of tetrahedra in $T \simeq S^{3}$ related by the symmetry give the same triangulation of $B^{3}$. The symmetry factors in $Z_{n_{3}, n_{1}}$ (tetrahedron) and $Z_{n_{3}, n_{1}}\left(S^{3}\right)$ compensate for the overcounting due to the symmetries, and Eq. (35) holds.

\section{$\S$ 6. Factorization}

In the previous section, we have shown that $\left\langle\Sigma^{(\alpha)}\right\rangle_{\mid S^{3}}$ gives a solution to the Schwinger-Dyson equation when $E\left(\Delta, \Delta_{0}\right)+1 \geq V\left(\Delta, \Delta_{0}\right)$ for any $\Delta$ on $\Sigma$ and when the 
surface $\Sigma$ in the equation is not degenerate. In this section, we will define a set of factorization conditions on $\left\langle\Sigma^{(\alpha)}\right\rangle_{\mid s^{3}}$. so that the Schwinger-Dyson equation becomes applicable even when it involves degenerate surfaces. We will then show that the Schwinger-Dyson equation combined with the factorization conditions has a unique solution. Thus they characterize the sum over triangulations $T$ such that $\mathscr{H}_{\Sigma}^{(\alpha)}$ $\times \cup_{\Sigma}(-T) \simeq S^{3}$.

Let us first consider a case when the surface $\Sigma$ is degenerate. When the pinching cycle is homologically trivial on $\Sigma$, the surface is actually separated into two components $\Sigma_{1}$ and $\Sigma_{2}$ touching to each other at the vertex: In this case, $T$ must also consist of two disconnected components $T_{1}$ and $T_{2}$ such that $\partial T_{i}=\Sigma_{i}(i=1,2)$. The Schwinger-Dyson equation remains applicable in this case if we supplement a condition that $\Delta$ in the summation on the right-hand side of Eq. (34) is restricted to be those on the same boundary component as $\Delta_{0}$ does. Especially when we can choose the meridians of the handlebody $\mathscr{H}_{\Sigma}^{(\alpha)}$ such that

$$
\left\{\alpha_{1}, \cdots, \alpha_{h(\Sigma)}\right\}=\left\{\alpha_{1}^{(1)}, \cdots, \alpha_{h\left(\Sigma_{1}\right)}^{(1)}\right\} \cup\left\{\alpha_{1}^{(2)}, \cdots, \alpha_{h\left(\Sigma_{2}\right)}^{(2)}\right\},
$$

where $\alpha_{i}^{(1)} \in \Sigma_{1}$ and $\alpha_{i}^{(2)} \in \Sigma_{2}$, the amplitude $\left.\left\langle\Sigma^{(\alpha)}\right\rangle\right|_{S^{3}}$ factorizes as

$$
\left\langle\Sigma^{(\alpha)}\right\rangle_{\mid S^{3}}=\left\langle\Sigma_{1}^{(\alpha(1)}\right\rangle_{\mid S^{3}}\left\langle\Sigma_{2}^{\alpha(2)}\right\rangle_{S^{3}} \text {. }
$$

In this case, it is obvious that $\Delta \in \Sigma_{i}$ should be imposed in Eq. (34) when $\Delta_{0} \in \Sigma_{i}$ (i $=1,2$ ).

It is instructive to compare this behavior of the amplitude with that of $\left\langle\Sigma^{(\alpha)}\right\rangle$ $=\left(\Phi^{(\alpha)}, \Psi_{\Sigma}\right)$. When $\Delta$ and $\Delta_{0}$ wrap around the homologically trivial cycle $\gamma$ and when the logitudes $\left\{\alpha_{i}\right\}$ are separated as in Eq. (36), $i_{\Delta, \Delta_{0}}$ defined by (25) gives a map from $V_{\Sigma}^{\text {(phys) }}$ into $V_{\Sigma_{1}}^{\text {(phys) }} \otimes V_{\Sigma_{2}}^{\text {(phys) }}$ as

$$
i\left[\Phi^{(\alpha)}\right]_{\Lambda, \Delta_{0}}=\Phi^{\left(\alpha^{(1)}\right)} \Phi^{(\alpha(2))} .
$$

The contribution of the degenerate surface $\Sigma_{\Delta, \Delta_{0}}$ to the Schwinger-Dyson equation is then

$$
\begin{aligned}
& \left\langle\Sigma_{\Delta, \Delta_{0}}^{(\alpha)}\right\rangle=\left\langle\sum_{1}^{\left(\alpha_{1}\right)} \sum_{2}^{\left(\alpha_{2}\right)}\right\rangle \\
& =\Lambda_{q}^{(1 / 2) n_{1}\left(\Sigma_{1}\right)+h\left(\Sigma_{1}\right)} \Lambda_{q}^{(1 / 2) n_{2}\left(\Sigma_{2}\right)+h\left(\Sigma_{2}\right)} \\
& \times \sum_{\left.j^{(1)}\right\}, \zeta^{(2)\}}} \Phi^{\left(\alpha^{(1)}\right)}\left(j^{(1)}\right) \Phi^{\left(\alpha^{(2)}\right)}\left(j^{(2)}\right) \Psi_{\Sigma_{1}+\Sigma_{2}}\left(j^{(1)}, j^{(2)}\right) \\
& =\left\langle\Sigma_{1}^{(\alpha(1))}\right\rangle\left\langle\Sigma_{2}^{(\alpha(2))}\right\rangle+\Lambda_{q} \sum_{T: \partial T=\Sigma_{1}+\Sigma_{2}}\left(\frac{C\left(\Sigma_{1}\right) C\left(\Sigma_{2}\right)}{C(T)}\right) g^{n_{3}(T)} \mu^{n_{1}(T)} \\
& \times \Lambda_{q}^{\chi(T)-(1 / 2) \chi\left(\Sigma_{1}\right)-(1 / 2) \chi\left(\Sigma_{2}\right)} I_{q}\left(\mathcal{H}_{\Sigma_{1}}^{\left(\alpha_{1}^{(1)}\right)} \cup_{\Sigma_{1}}(-T) \cup_{\Sigma_{2}} \mathcal{H}_{\Sigma_{2}}^{\left(q^{(2)}\right)}\right) .
\end{aligned}
$$

In the first term on the right-hand side, the sum is over simplicial complexes consisting of two disconnected components $T_{1}$ and $T_{2}$ each of which is attached to $\Sigma_{1}$ and $\Sigma_{2}$ respectively, and the second term is a sum over connected simplicial complexes. We see that the second term is multiplied by the extra-factor of $\Lambda_{q}$. This is interpreted as follows. If $T$ remains connected after pinching around the cycle $\gamma, T$ should 
contain a homotopy cycle linking with $\gamma$. The extra-factor $\Lambda_{q}$ is associated with this homotopy cycle. The factorization condition may be regarded as the $q \rightarrow 0$ limit of this equation for $\left\langle\Sigma^{(\alpha)}\right\rangle$ where the second term is to be suppressed by the factor of $\Lambda_{q}$.

When the surface $\Sigma$ is degenerate around a homologically non-trivial cycle, the sum over $\Delta$ in the Schwinger-Dyson equation runs over all triangles on $\Sigma_{\Delta_{0}}$ as far as $\Delta_{0}$ is not on the degeneration point. When one of the vertices of $\Delta_{0}$ is on the degeneration point, we must supplement the following rule: The sum over triangles in the Schwinger-Dyson equation should not include those in the neighborhood of $\Delta_{0}$ and on the other side of the degeneration point across the degenerating cycle. The reason for this is the following. If we take a triangle $\Delta^{\prime}$ which shares the degeneration point with $\Delta_{0}$ and which is on the other side of the point, the identification of $\Delta^{\prime}$ and $\Delta_{0}$ does not change the Euler number of $\Sigma$. The number of triangles in $T$, on the other hand, decreases by one if $\Delta^{\prime}$ and $\Delta_{0}$ were identified in $T$ and removed from $T$. Thus the equality $\chi(T)=(1 / 2) \chi(\Sigma)$ cannot be sustained. On the other hand, if we take $\Delta$ to be other than those triangles, there is a triangulation $T$ such that $\mathscr{H}_{\Sigma}^{(\alpha)}$ $\times \cup(-T) \simeq S^{3}$ and $\Delta$ and $\Delta_{0}$ are identified. Thus the Schwinger-Dyson equation holds with this supplementary condition.

So far, we have examined the situation when $\Sigma$ is already degenerate. When $V\left(\Delta, \Delta_{0}\right) \geq E\left(\Delta, \Delta_{0}\right)+2$, the triangles $\Delta$ and $\Delta_{0}$ wrap around a cycle $\gamma$ on $\Sigma$ and the surface will develop additional degeneration if we identify $\Delta$ and $\Delta_{0}$ and remove them. If it is possible to construct a triangulation $T$ in which the two triangles $\Delta$ and $\Delta_{0}$ are attached to each other, $T$ should be counted in $\left\langle\Sigma^{(\alpha)}\right\rangle_{S^{3}}$. The Schwinger-Dyson equation (26) derived for non-degenerate surfaces becomes applicable in this case if we take into account the contribution of the degenerate surface $\Sigma_{\Delta, \Delta_{0}}$. On the other hand, if there is no such triangulation in which $\Delta$ is identified with $\Delta_{0}$, the surface $\Sigma_{\Delta, \Delta_{0}}$ should not be included in the Schwinger-Dyson equation.

When the cycle $\gamma$ is homologically trivial, it is always possible to construct a triangulation $T$ in which $\Delta$ and $\Delta_{0}$ are identified. Thus the term $\left\langle\Sigma_{\Delta, \Delta_{0}}^{\alpha}\right\rangle$ should be included in the Schwinger-Dyson equation. In this case, the surface $\Sigma_{\Delta, \Delta_{0}}$ is pinched around at the common vertex of $\Delta$ and $\Delta_{0}$, and the Schwinger-Dyson equation can be iterated with the supplementary conditions introduced in the above.

On the other hand, when the cycle $\gamma$ is homologically non-trivial on $\Sigma$, the triangles $\Delta$ and $\Delta_{0}$ wrap around a handle on $\Sigma$. In this case, the handle is pinched on the surface $\Sigma_{\Delta, \Delta_{0}}$. If there is a triangulation $T$ in which the triangles $\Delta$ and $\Delta_{0}$ are identified with each other, there must be a disc in $T$ whose boundary is the cycle $\gamma$. After removing the triangle $\Delta=\Delta_{0}$ from $T$, we obtain $T^{\prime}$ which gives a triangulation of a handlebody whose boundary is $\Sigma_{\Delta, \Delta_{0}}$. The surface $\Sigma_{\Delta, \Delta_{0}}$ is pinched at the common vertex of $\Delta$ and $\Delta_{0}$. The complement of $T^{\prime}$ in $S^{3}$ should also be a handlebody $\mathscr{H}_{\Sigma_{\Delta, \Delta_{0}}}^{(\alpha)}$ which is pinched around the cycle $\gamma$. The process $\mathcal{H}_{\Sigma}^{(\alpha)} \cup(-T) \rightarrow \mathcal{H}_{\Sigma_{\Delta, \Delta 0}}^{(\alpha)} \cup\left(-T^{\prime}\right)$ then gives the reduction of the Heegaard splitting of $S^{3}$ which is the inverse of the stabilization. $^{38)}$ For this to be possible, there must be a disc in the handlebody $\mathscr{H}_{\Sigma}^{(\alpha)}$ whose boundary is on $\dot{\Sigma}$ and intersects once with $\gamma$ transversely. If this is the case, we can choose the meridians of $\mathscr{H}_{\Sigma}^{(\alpha)}$ so that $\alpha_{1} \cdot \gamma=1$ and $\alpha_{i} \cdot \gamma=0(i=2, \cdots, h(\Sigma))$. The interior of $\mathcal{H}_{\Sigma_{\Delta, \Delta_{0}}}^{\left(\alpha_{1}, \cdots, \alpha_{h(\Sigma)}\right)}$ is then homeomorphic to that of $\mathcal{H}_{\Sigma_{\Delta, \Delta_{0}}}^{\left(\alpha_{2}, \cdots, \alpha_{h(\Sigma)}\right)}$, where $\Sigma_{\Delta, \Delta_{0}}^{\prime}$. is 
obtained from $\Sigma_{\Delta, \Delta_{0}}$ by splitting the common vertex of $\Delta$ and $\Delta_{0}$ into two so that the pinched handle is removed; $h\left(\Sigma_{\Delta, \Delta_{0}}^{\prime}\right) h\left(\Sigma_{\Delta, \Delta^{\prime}}\right)-1$. In this case, a term $\left\langle\Sigma_{\Delta, \Delta_{0}}^{\prime(\alpha)}\right\rangle_{M}$ must be included in the Schwinger-Dyson equation. Conversely, if it is not possible to find such a disc in $\mathcal{H}_{\Sigma}^{(\alpha)}$, there is no triangulation in which $\Delta$ and $\Delta_{0}$ are identified with each other. In this case, there should be no term in the Schwinger-Dyson equation corresponding to the surface $\Sigma_{\Delta, \Delta_{0}}$.

With these supplementary conditions, we can apply the Schwinger-Dyson equation even when $\Sigma$ is degenerate or when there is a triangle $\Delta$ such that $V\left(\Delta, \Delta_{0}\right) \geq E(\Delta$, $\left.\Delta_{0}\right)+2$. As in the case of the matrix model, we can show that the Schwinger-Dyson equation combined with these conditions can be solved inductively. The SchwingerDyson equation expressed as in Eq. (34) is a recurrence relation for $Z_{n_{3}, n_{1}}(\alpha ; \Sigma)$ along the bibliographical ordering: $\left(n_{3}, \Sigma\right)>\left(n_{3}^{\prime}, \Sigma^{\prime}\right)$ if $n_{3}>n_{3}^{\prime}$ or $n_{3}=n_{3}^{\prime}$ and $n_{2}(\Sigma)>n_{2}\left(\Sigma^{\prime}\right)$. Because of the factorization conditions, we can trace back the Schwinger-Dyson equation to the expectation value of the null surface $\langle 1\rangle$ which is normalized to be one. Thus the Schwinger-Dyson equation combined with the factorization conditions has a unique solution. The solution gives the sum over triangulations of handlebodies, and it is related to $Z_{n_{3}, n_{1}}\left(S^{3}\right)$ as in Eq. (35).

\section{Acknowledgements}

I would like to thank J. Ambjфrn, B. Durhuus, T. Eguchi, A. Jevicki and T. Matsumoto for discussions and comments. I would also like to thank M. Atiyah and P. Goddard for their hospitality at the Isaac Newton Institute for Mathematical Sciences, University of Cambridge, where part of this work was done. This research is supported in part by the Grant-in-Aid for Scientific Research on Priority Areas 231 "Infinite Analysis" from the Minstry of Education, Science and Culture of Japan.

\section{References}

1) T. Regge, Nuovo Cim. 19 (1961), 558.

2) For a review on recent numerical results in this approach, see H. Hamber, Preprint UCI-Th-91-36 (1991).

3) W. T. Tutte, Can. J. Math. 14 (1962), 21.

4) E. Brézin, C. Itzykson, G. Parisi and J.-B. Zuber, Commun. Math. Phys. 59 (1978), 35.

5) T. Eguchi and H. Kawai, Phys. Lett. 114B (1982), 247.

6) F. David, Nucl. Phys. B257 (1985), 45.

7) V. A. Kazakov, Phys. Lett. 150B (1985), 282.

8) D. Bessis, C. Itzykson and J.-B. Zuber, Adv. Appl. Math. 1 (1980), 109.

9) I. Kostov and M. L. Metha, Phys. Lett. 189B (1987), 118.

10) V. G. Knizhnik, A. M. Polyakov and A. B. Zamolodchikov, Mod. Phys. Lett. A3 (1988), 819.

11) F. David, Mod. Phys. Lett. A3 (1988), 1651.

J. Distler and H. Kawai, Nucl. Phys. B321 (1989), 509.

12) M. Douglas and S. Shenker, Nucl. Phys. B335 (1990), 635.

D. J. Gross and A. A. Migdal, Phys. Rev. Lett. 64 (1990), 127.

E. Brézin and V. A. Kazakov, Phys. Lett. B236 (1990), 144.

13) E. Witten, Nucl. Phys. B340 (1990), 281.

R. Dijkgraaf and E. Witten, Nucl. Phys B342 (1990), 342.

14) R. Dijkgraaf, E. Verlinde and H. Verlinde, Nucl. Phys. B348 (1991), 435.

E. Verlinde and H. Verlinde, Nucl. Phys B348 (1991), 457.

15) M. Fukuma, H. Kawai and R. Nakayama, Int. J. Mod. Phys. A6 (1991), 1385.

16) G. Moore, N: Seiberg and M. Staudacher, Nucl. Phys B362 (1991), 665.

G. Moore and N. Seiberg, Int. J. Mod. Phys. A7 (1991), 190. 
17) J. Ambjørn and S. Varsted, Phys. Lett. 266B (1991), 285.

18) M. E. Agishtein and A. A. Migdal, Mod. Phys. Lett. A6 (1991), 1863.

19) D. V. Boulatov and A. Krywicki, Mod. Phys. Lett. A6 (1991), 3005.

20) J. Ambjørn and S. Varsted, Peprint NBI-HE-91-45 (19.91).

21) J. Ambjørn, D. V. Boulatov, A. Krywicki and S. Varsted, Preprint NBI-HE-91-46 (1991).

22) J. Ambjørn, B. Durhuus and T. Jonsson, Mod. Phys. Lett A6 (1991), 1133.

23) N. Sasakura, Mod. Phys. Lett. A6 (1991), 2613.

24) M. Godfrey and M. Gross, Phys. Rev. D43 (1992), R1749.

25) D. V. Boulatov, Preprint SPhT/92-017 (1992).

26) V. G. Turaev and O. Y. Viro, Topology 31 (1992), 865.

27) S. R. Wadia, Phys. Rev. D24 (1981), 970.

28) A. A. Migdal, Phys. Rep. 102 (1983), 199.

29) A. N. Kirillov and N. Yu. Reshetikhin, in Infinite Dimensional Lie Algebras and Groups, ed. V. G. Kac (World Scientific, 1989).

30) V. G. Turaev, C. R. Acad. Sci. Paris, t. 313, Série I (1991), 395; "Topology of Shadow," Preprint (1991).

31) K. Walker, "On Witten's 3-Manifold Invariants," Preprint (1990).

32) E. Witten, Commun. Math. Phys. 121 (1983), 351,

N. Y. Reshetikhin and V. G. Turaev, Invent. Math. 103 (1991), 547.

33) H. Ooguri and N. Sasakura, Mod. Phys. Lett. A6 (1991), 3591.

34) H. Ooguri, Nucl. Phys. B382 (1992), 276.

35) F. Archer and R. Williams, Phys. Lett. B273 (1991), 438.

36) S. Mizoguchi and T. Tada, Phys. Rev. Lett. 68 (1992), 1795.

37) T. Kohno, Topology 31 (1992), 203.

38) F. Waldhausen, Topology 7 (1968), 195. 